\title{
Tunable metasurfaces (Presentation Recording)
}

\author{
Harry A. Atwater, California Institute of Technology (United States)
}

\begin{abstract}
Metasurfaces composed of sub-wavelength artificial structures show promise for extraordinary light-manipulation and development of ultrathin optical components such as lenses, wave plates, orbital angular detection, and holograms over a broad range of the electromagnetic spectrum. However structures developed to date do not allow for post-fabrication control of antenna properties. We have investigated the integration of the transparent conductor indium tin oxide (ITO) active elements to realize gate-tunable phased arrays of subwavelength patch antenna in a metasurface configuration to enable gate tunable permittivity. The magnetic dipole resonance of each patch antenna interacts with the carrier density-dependent permittivity resonance of the ITO to enable phase and amplitude tunability. Operation of patch antennas and beam steering phased arrays will be discussed.
\end{abstract}

View presentation video on SPIE's Digital Library:

http://dx.doi.org/10.1117/12.2191641.4519316172001 